\title{
Acoustic oscillations in solar and stellar flaring loops
}

\author{
V. M. Nakariakov ${ }^{1}$, D. Tsiklauri ${ }^{1, \star}$, A. Kelly ${ }^{1}$, T. D. Arber ${ }^{1}$, and M. J. Aschwanden ${ }^{2}$ \\ 1 Physics Department, University of Warwick, Coventry, CV4 7AL, UK \\ 2 Lockheed Martin, Advanced Technology Center Solar \& Astrophysics Lab, Dept. L9-41, Bldg. 252, 3251 Hanover Street, \\ Palo Alto, CA 94304, USA
}

Received 20 November 2003 / Accepted 11 December 2003

\begin{abstract}
Evolution of a coronal loop in response to an impulsive energy release is numerically modelled. It is shown that the loop density evolution curves exhibit quasi-periodic perturbations with the periods given approximately by the ratio of the loop length to the average sound speed, associated with the second standing harmonics of an acoustic wave. The density perturbations have a maximum near the loop apex. The corresponding field-aligned flows have a node near the apex. We suggest that the quasi-periodic pulsations with periods in the range 10-300 s, frequently observed in flaring coronal loops in the radio, visible light and X-ray bands, may be produced by the second standing harmonic of the acoustic mode.
\end{abstract}

Key words. Sun: flares - Sun: oscillations - Sun: corona - stars: flare - stars: oscillations - stars: coronae

\section{Introduction}

Wave activity of the solar corona attracts attention in relation with coronal heating and solar wind acceleration problems, and as an efficient tool for MHD coronal seismology (e.g. Nakariakov 2003). The observational evidence of coronal waves and oscillations is abundant. Low period coronal oscillations, in the range between a few seconds to several minutes, are believed to be associated with magnetohydrodynamic waves. In particular, propagating slow magnetoacoustic waves have been identified in polar plumes (Ofman et al. 1999) and over loop footpoints (Nakariakov et al. 2000; Tsiklauri \& Nakariakov 2001), and standing global slow modes in loops (Ofman \& Wang 2002). The majority of confidently interpreted examples of the coronal wave activity has been found in the EUV coronal emission.

Observations in other spectral windows, in particular in the radio band, also demonstrate various kinds of oscillations (e.g., the quasi-periodic pulsations, or QPP, see Aschwanden 1987 for a review), usually with periods from a few seconds to several tens of seconds. It is commonly accepted that the waves with periods about several seconds may be produced by either propagating or standing sausage modes (Roberts et al. 1984; Nakariakov et al. 2003; Aschwanden et al. 2004). However, it is known that the existence of the non-leaky sausage mode cut-off imposes an upper limit on the possible wave periods: waves with the periods longer than the cut-off period cannot be

Send offprint requests to: V. M. Nakariakov, e-mail: valery@astro.warwick.ac.uk

* Present address: Joule Physics Laboratory, School of Computing, Science and Engineering, University of Salford, Salford, M5 4WT, UK. trapped in the loops. For a magnetic cylinder of the radius $a$, the cut-off period $P_{\mathrm{c}}$ is estimated (see, e.g. Roberts et al. 1984) as

$$
P_{\mathrm{c}} \approx \frac{2.62 a}{C_{\mathrm{Ae}}} \sqrt{\frac{C_{\mathrm{Ae}}^{2}-C_{\mathrm{A} 0}^{2}}{C_{\mathrm{s} 0}^{2}+C_{\mathrm{A} 0}^{2}}},
$$

where $C_{\mathrm{s} 0}$ is the sound speed in the loop, and $C_{\mathrm{A} 0}$ and $C_{\mathrm{Ae}}$ are the Alfvén speeds inside and outside the slab, respectively. For example, for typical flaring loop parameters $a=6 \mathrm{Mm}$, $C_{\mathrm{A} 0}=1 \mathrm{Mm} \mathrm{s}^{-1}, C_{\mathrm{Ae}}=3 C_{\mathrm{A} 0}$ and $C_{\mathrm{s} 0}=0.5 \mathrm{Mm} \mathrm{s}^{-1}$, the cutoff period is about $15 \mathrm{~s}$, and it is difficult to make it greater than $20 \mathrm{~s}$. However, X-ray band observations often give much longer periodicities, frequently in association with a flare. For example, periodicities from $20 \mathrm{~s}$ to $25 \mathrm{~min}$ have been presented by Harrison (1987), McKenzie \& Mullan (1997) and Terekhov et al. (2002). Similar periodicities have been observed in the decimeter and microwave bands. In particular, Wang \& Xie (2000) observed QPP with the periods of about $50 \mathrm{~s}$ at 1.42 and $2 \mathrm{GHz}$ (in association with an M4.4 X-ray flare). The coincidence of QPP periods observed in the X-ray and in radio bands is not a surprise, as the higher frequency radio bursts are found to correlate very well with X-ray bursts (e.g., Benz \& Kane 1986). Moreover, pulsations with the periods significantly greater than the estimated sausage mode cut-off period have been found in both hard X-ray and microwave bands simultaneously (e.g. Fu et al. 1996; Tian et al. 1999).

Similar oscillations, with the period of $220 \mathrm{~s}$, have recently been observed in the white-light emission associated with stellar flaring loops (Mathioudakis et al. 2003). Possibly, the $160 \mathrm{~s}$ periodic oscillations observed by Houdebine et al. (1993) during a flare on Ad-Leonis and 26 and 13 s coherent oscillations 
observed by Zhilyaev et al. (2000) in EV Lac flares have the same nature.

A possible interpretation of these medium period QPPs may be connected with kink or torsional modes (Zaitsev \& Stepanov 1989). However, these modes are practically incompressible and, in the case of a small amplitude, the produced perturbation of the magnetic field is also very weak. (E.g., the direct observations of kink modes in EUV, Aschwanden et al. 1999; Nakariakov et al. 1999.) Thus, it is not simple to link these weak perturbations of the magnetic field with observed QPPs.

In this study, we suggest an alternative mechanism for the generation of long-period QPPs. We demonstrate that in a coronal loop an impulsive energy release generates the second spatial harmonics of the acoustic mode. These oscillations are of high quality and do not experience dissipation. This mode, producing noticeable perturbations of the loop density and generating field-aligned flows, is shown to be responsible for QPP with medium and long periods.

\section{The model}

We describe plasma dynamics in a coronal loop by solving numerically 1D radiative hydrodynamic equations (infinite magnetic field approximation) that resemble closely the Naval Research Laboratory Solar Flux Tube Model (SOLFTM, Mariska 1987). The numerical code that we use is a 1D version of Lagrangian Re-map code (Arber et al. 2001) with the radiative loss limiters. As in SOLFTM the coronal loop is connected with a dense, cold $\left(10^{4} \mathrm{~K}\right), 5 \mathrm{Mm}$ thick plasma region, that mimics the chromosphere, and which because of its large density provides sufficient amount of matter to fill the loop during the flare. The model includes: the effects of gravitational stratification, heat conduction, radiative losses, added external heat input, the presence of helium, non-linearity, and Braginskii bulk viscosity. For the radiative loss function we use the form given in Rosner et al. (1978) extended to a wider temperature range (Peres et al. 1982; Priest 1982).

We performed a series of numerical runs simulating the response of the loop to a flare-like impulsive heat deposition at a chosen location. The heat deposition was modelled by the function

$$
\begin{aligned}
E_{\mathrm{H}}(s, t) & =E_{\mathrm{S}}(s) E_{\mathrm{T}}(t) \\
& =E_{0} \exp \left(-\frac{\left(s-s_{0}\right)^{2}}{2 \sigma_{\mathrm{s}}^{2}}\right)\left[1+Q_{\mathrm{p}} \exp \left(-\frac{\left(t-t_{\mathrm{p}}\right)^{2}}{2 \sigma_{\mathrm{t}}^{2}}\right)\right],
\end{aligned}
$$

where, $E_{\mathrm{S}}(s)$ and $E_{\mathrm{T}}(t)$ were spatial and temporal parts of the heating function. $E_{0}$ is the amplitude in units of $\mathrm{erg} \mathrm{cm}^{-3} \mathrm{~s}^{-1} ; s_{0}$ is the position of the maximum heat deposition; $\sigma_{\mathrm{s}}$ is the heat deposition localization scale. Function (2) allows us to model spatial and temporal profiles of the heat deposition separately. As it is discussed below, the results are weakly dependent upon the particular choice of this function. The only restriction is the spatial and temporal characteristic scales to be shorter than the loop length and the acoustic transit time along the loop, respectively.

In the numerical runs presented here, the size of the heat deposition region is $\sigma_{\mathrm{s}}=7 \mathrm{Mm}$. The flare peak time is fixed
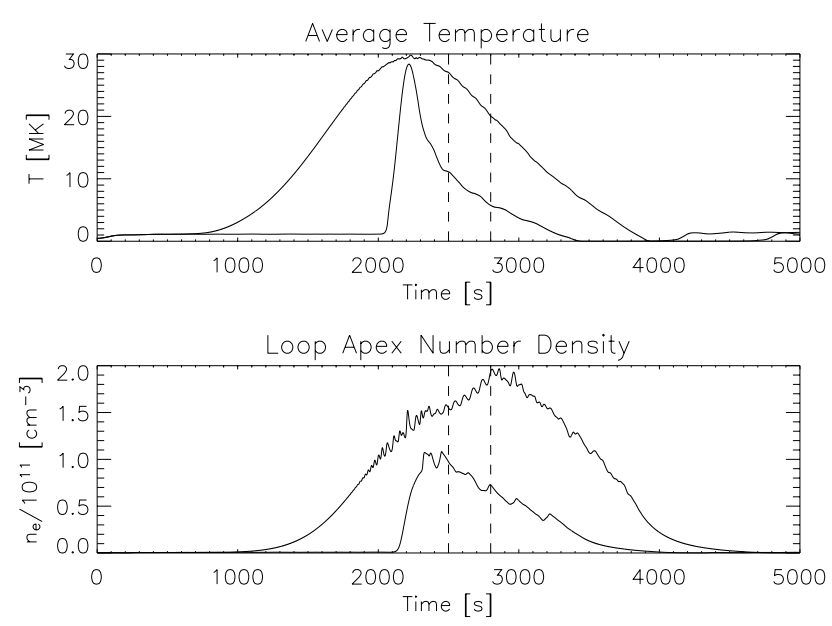

Fig. 1. Time evolution of the average temperature (upper panel) and the number density at apex (lower panel) in the flaring loop in the case of apex heating. The upper curves correspond to longer flares and the lower to the shorter flares. The vertical straight lines show the time interval of particularly distinct oscillations on the upper curve.

in at $2200 \mathrm{~s}$. The heat deposition duration, $\sigma_{\mathrm{t}}$, is variable, from $42 \mathrm{~s}$ to $333 \mathrm{~s}$. The heating amplitude $Q_{\mathrm{p}}$ is fixed at $2 \times 10^{4}$, while $E_{0}$ is fixed at $0.004 \mathrm{erg} \mathrm{cm}^{-3} \mathrm{~s}^{-1}$. The loop length is taken to be $55 \mathrm{Mm}$. We keep the coronal part of the loop initially at a temperature of $1 \mathrm{MK}$ and at a mass density of $\rho=\mu m_{\mathrm{p}} n_{\mathrm{e}}=6.6 \times 10^{-16} \mathrm{~g} \mathrm{~cm}^{-3}$ (at the loop apex), for a heliumto-hydrogen number density ratio of 0.05 , i.e., with the mean molecular weight $\mu=1.1$. This corresponds to an electron density of $n_{\mathrm{e}}=3.6 \times 10^{8} \mathrm{~cm}^{-3}$. In our approach, specific initial conditions in region connecting corona to the chromosphere and chromosphere itself have little or no effect on the corona dynamics as they are rapidly modified, self-consistently, according to the radiative hydrodynamics equations. Resolution in all numerical runs was fixed at 1000 grid points, which were distributed non-uniformly in order to properly resolve strong gradients in the transition region. Even tripling the number of grid points does not alter the numerical results, thus assuring full convergence of the simulation.

\section{Acoustic second standing harmonics}

The typical loop evolution is shown in Fig. 1. The impulsively heated loop responds with an increase of the temperature. The particular response is determined by the duration of the heat deposition $\sigma_{\mathrm{t}}$. In Fig. 1 the cases of $\sigma_{\mathrm{t}}=42 \mathrm{~s}$ and $333 \mathrm{~s}$ are presented. The chromospheric evaporation increases loop density with up-flows of the order of a hundred $\mathrm{km} \mathrm{s}^{-1}$. Then, during the peak of the flare, the combined action of the heat input and the conductive and radiate losses, yields an oscillatory flow pattern with typical amplitudes of up to few tens of $\mathrm{km} \mathrm{s}^{-1}$. In the cooling phase, the matter drains out of the loop exhibiting oscillations; and the loop finally reaches an equilibrium.

In the context of this study, the most interesting feature in Fig. 1 is the clear quasi-periodic oscillations of the density time-profiles, especially about and after the peak of the flare. This behaviour closely resembles the radio and X-ray flaring light-curves (see the citations in Introduction). 
Typical time-distance plots of the evolution of the density and the velocity are shown in Fig. 2. Here, the slow, in comparison with the oscillation period, aperiodic variations of the density and the velocity are subtracted. The velocity perturbation clearly demonstrates the node near the apex, while the density perturbation is maximal there (anti-node). This behaviour is consistent with the second standing acoustic mode,

$$
\begin{aligned}
& V_{x}(s, t)=A \cos \left(\frac{\pi C_{\mathrm{s}}}{L} t\right) \sin \left(\frac{\pi}{L} s\right), \\
& \rho(s, t)=-\frac{A \rho_{0}}{C_{\mathrm{s}}} \sin \left(\frac{\pi C_{\mathrm{s}}}{L} t\right) \cos \left(\frac{\pi}{L} s\right),
\end{aligned}
$$

where $C_{\mathrm{s}}$ is a speed of sound, $A$ is wave amplitude, $L$ is loop length, and $s$ is a distance along the loop with the zero at the loop top.

The top panel of Fig. 3 shows characteristic time signatures of the density and velocity oscillations at two points symmetric with respect to the loop apex. Depending upon the leg of the loop, the phase shift between the density and the velocity oscillations is plus or minus quarter period, as it is expected for the second standing acoustic harmonic (see Eq. (3)). Perturbations of the density are in phase at both points of measurement. The oscillations are quasi-harmonic, with a well-defined period. In the discussed case, the period of the oscillation is $64 \mathrm{~s}$. According to Eq. (3), the period of the second spatial harmonic should be $P=L / C_{\mathrm{s}}$. The practical formula for the determination of the oscillation period is

$P / \mathrm{s} \approx 6.7 \times(L / \mathrm{Mm}) / \sqrt{(T / \mathrm{MK})}$

where $T$ is the average temperature in the loop. (This expression is a particular case of the general formula suggested by Roberts et al. 1984, who pointed out that the second standing harmonic could likely be excited in coronal loops.) Substituting the loop length $L=55 \mathrm{Mm}$ (see Fig. 2) and the average temperature, $25 \mathrm{MK}$ (see top left panel in Fig. 1 in the range of 2500-2800 s), we obtain $73.7 \mathrm{~s}$, which is reasonably close to the result of the simulations. The discrepancy of about $15 \%$ is explained by the fact that the actual reflection points are situated over the loop footpoints (see Fig. 2) because of stratification. This reduces slightly the effective length of the loop and, consequently the resonant period of the mode. However, as the uncertainty in the observational determination of the loop length is comparable with this error, formula (5) provides a satisfactory estimation of the oscillation period.

\section{Conclusions}

We suggest that the second standing acoustic mode may be responsible for QPP with periods of about 10-300 s (estimated by Eq. (3)) observed in solar and stellar flare light curves. This mechanism is similar to the interpretation of coronal loop oscillations observed with SUMER, proposed by Ofman \& Wang (2002). The main new element in our study is connected with the mode excitation. We demonstrate that the second standing acoustic harmonic appears as a natural response of the loop to an impulsive energy deposition. The SUMER oscillations are likely to be associated with some other excitation mechanism,
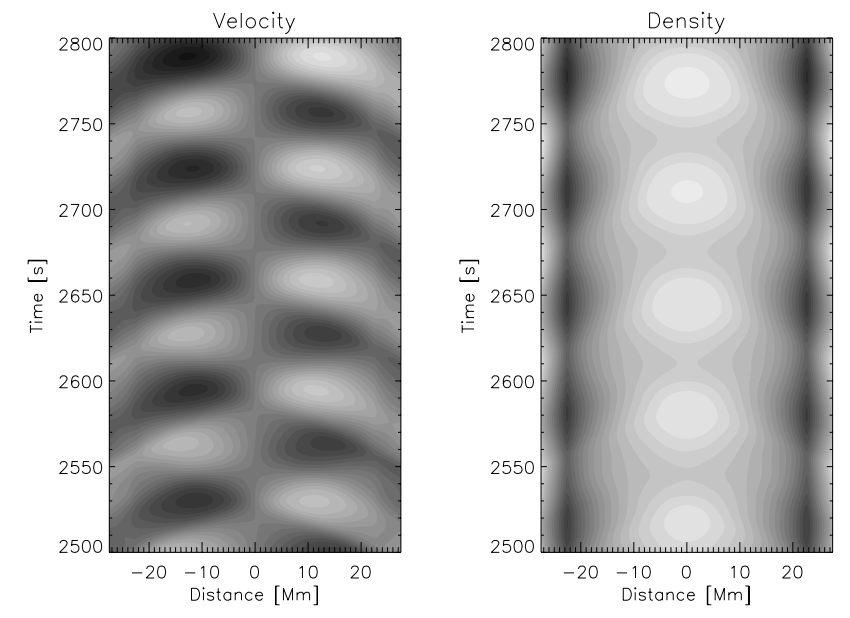

Fig. 2. The time-distance plots of perturbations of the velocity and density, after subtraction of the slow evolution of the background, for the time interval highlighted for the upper curve in Fig. 1.
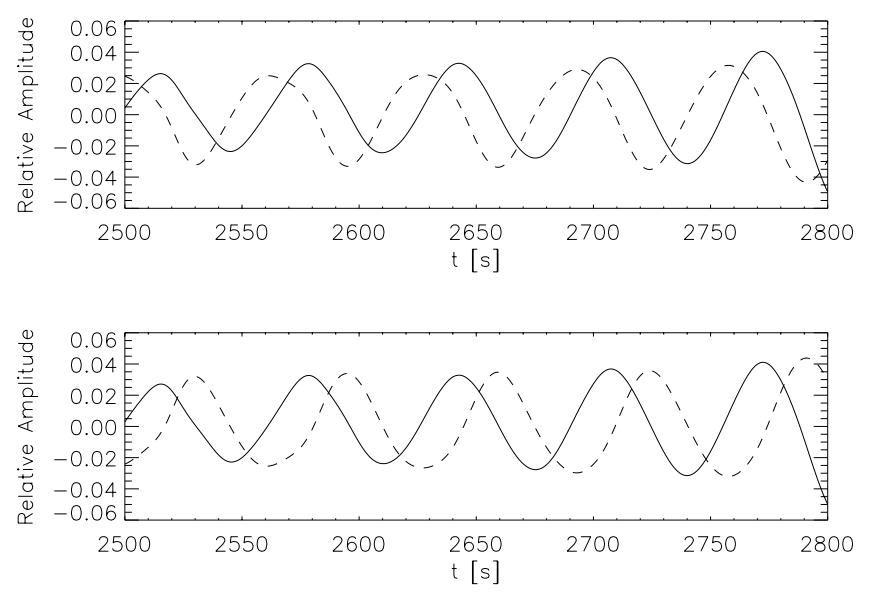

Fig. 3. Time signatures of the velocity and density perturbations outputted at the points $-6 \mathrm{Mm}$ (top panel) and $+6 \mathrm{Mm}$ (bottom panel) of the loop length for the same time interval as Fig. 2. The solid curve shows the plasma number density in units of $10^{11} \mathrm{~cm}^{-3}$. The dashed line gives the velocity normalized to $400 \mathrm{~km} \mathrm{~s}^{-1}$. Here, the slow variations of the density and the velocity are subtracted.

as only a small fraction of SUMER oscillations are observed in association with solar flares (Wang et al. 2003).

Traditionally, the acoustic wave interpretation was excluded as these waves were supposed to be highly dissipative. However, numerical simulations discussed above, as well as recently gained abundant observational evidence of the presence of acoustic waves in the solar corona, suggest that the observed periodicities can be associated with this mode. The physical mechanism responsible for the induction of the quasi-periodic pulsations can be understood in terms of auto-oscillations generated by an electric-circuit generator. Indeed, the physical system modelled here contains all the necessary ingredients of a generator: the DC power supply (thermal instability), the nonlinear element (the plasma) and the resonator (the loop). This may explain why the oscillations may be observed to be dissipationless. However, proper analytical theory of the excitation of this mode is still to be developed. 
The oscillation period is determined by the ratio of the loop length and the average sound speed in the loop. The typical amplitude of the density and velocity perturbations is $2-10 \%$ of the background. This value is consistent with observed amplitudes of X-ray QPPs (McKenzie \& Mullan 1997). However, the observed amplitudes of radioband QPPs are sometimes higher. This discrepancy would be resolved by taking into account the specific mechanism responsible for the modulation of radio emission, and the line-of-sight angle (e.g. Cooper et al. 2003). In certain condition, the modulation mechanism can amplify the pulsations up to the observable level.

The model developed here is quite approximate, as it does not take into account two-dimensional MHD effects such as centrifugal force and the perturbation of the loop cross-section. However, we believe that the neglected effects do not change the qualitative picture described here and that Eq. (5) gives a correct estimation for the oscillation period.

Finally, we would like to emphasize that the generation of the second spatial harmonic of an acoustic wave, by means of which we try to explain the observed quasi-periodic oscillations in flaring loops, is a consistent feature, seen for a wide range of physical parameters, including the case of nonsymmetric heating functions. More detailed analysis will be presented elsewhere.

Acknowledgements. Numerical calculations of this work were performed using the PPARC funded Compaq MHD Cluster at St Andrews and Astro-Sun cluster at Warwick. This work was supported in part by PPARC (DT) and EPSRC (AK). The authors are grateful to the referee, Mihalis Mathioudakis, for valuable comments.

\section{References}

Arber, T. D., Longbottom, A. W., Gerrard, C. L., \& Milne, A. M. 2001, J. Comput. Phys., 171, 151

Aschwanden, M. J. 1987, Sol. Phys., 111, 113

Aschwanden, M. J., Fletcher, L., Schrijver, C. J., \& Alexander, D. 1999, ApJ, 520, 880
Aschwanden, M. J., Nakariakov, V. M., \& Melnikov, V. F. 2004, ApJ, in press

Benz, A. O., \& Kane, S. R. 1986, Sol. Phys., 104, 179

Cooper, F. C., Nakariakov, V. M., \& Williams, D. R. 2003, A\&A, 409, 325

Fu, Q.-J., Liu, Y.-Y., \& Li, C.-S. 1996, Chin. Astron. Astrophys., 20, 487

Harrison, R. A. 1987, A\&A, 182, 337

Houdebine, E. R., Foing, B. H., Doyle, J. G., \& Rodono, M. 1993, A\&A, 274, 245

Mariska, J. T. 1987, ApJ, 319, 465

Mathioudakis, M., Seiradakis, J. H., Williams, D. R., et al. 2003, A\&A, 403, 1101

McKenzie, D. E., \& Mullan, D. J. 1997, Sol. Phys., 176, 127

Nakariakov, V. M. 2003, in Dynamic Sun, ed. B. Dwivedi, CUP

Nakariakov, V. M., Ofman, L., DeLuca, E. E., Roberts, B., \& Davila, J. M. 1999, Science, 285, 862

Nakariakov, V. M., Verwichte, E., Berghmans, D., \& Robbrecht, E. 2000, A\&A, 362, 1151

Nakariakov, V. M., Melnikov, V. F., \& Reznikova, V. E. 2003, A\&A, 412, L7

Ofman, L., Nakariakov, V. M., \& DeForest, C. E. 1999, ApJ, 514, 441

Ofman, L., \& Wang, T. J. 2002, ApJ, 580, L85

Peres, G., Serio, S., Vaiana, G. S., \& Rosner, R. 1982, ApJ, 252, 791

Priest, E. R. 1982, Solar Magnetohydrodynamics (Dordrecht: D. Reidel Publ. Comp.)

Roberts, B., Edwin, P. M., \& Benz, A. O. 1984, ApJ, 279, 857

Rosner, R., Tucker, W. H., \& Vaiana, G. S. 1978, ApJ, 220, 643

Terekhov, O. V., Shevchenko, A. V., Kuz'min, A. G., et al. 2002, Astron. Lett., 28, 397

Tian, D.-W., Gao, Z.-M., \& Fu, Q.-J. 1999, Chin. Astron. Astrophys., 23, 208

Tsiklauri, D., \& Nakariakov, V. M. 2001, A\&A, 379, 1106

Wang, M., \& Xie, R. X. 2000, Chin. Astron. Astrophys., 24, 95

Wang, T. J., Solanki, S. K., Curdt, W., et al. 2003, A\&A, 406, 1105

Zaitsev, V. V., \& Stepanov, A. V. 1989, Sov. Astron. Lett., 15, 66

Zhilyaev, B. E., Romanyuk, Y. O., Verlyuk, I. A., et al. 2000, A\&A, 364,641 